\title{
GEOCHEMICAL CHARACTERISTICS OF THE MAFIC ENCLAVES AND THEIR HOSTS FROM NEOGENE ERENLERDAGI VOLCANITES, AROUND YATAGAN VILLAGE AND SAĞLIK TOWN (KONYA), CENTRAL TURKEY
}

\author{
Koçak K. ${ }^{1}$ \\ ${ }^{1}$ Selcuk University Konya, TURKEY, Department of Geology, 54124, Konya, TURKEY, \\ kkocak@yahoo.com,kkocak@selcuk.edu.tr
}

\begin{abstract}
Late Miocene to Pliocene volcanism is represented by development of lava domes, nuée ardentes and pyroclastic fall and flow (ignimbrites) deposits in the WSW and NW of Konya city. The lava dome contains various mafic microgranular enclaves (MMEs), which have various size (a few cm to a few meters), shape (ellipse/sphere to rounded), with a well-developed chilly zone. The MMEs samples are situated on mostly basaltic andesite and andesite, and a few MME samples on basaltic trachyandesite area while the host rocks are concentrated on dacite and andesite areas. The felsic samples have more fractionated chondrite-normalised REE pattern ( $\mathrm{La} / \mathrm{Yb}_{N}$ : 9.5-18.1) than MMEs (6.7-16.0) ones, but both have slightly developed negative Eu anomaly (Eu/Eu*: 0.67-0.89 in felsic rocks, 0.68-0.87 in MMEs). In primitive mantlenormalized spider diagram, the MMEs and felsic rocks have negative $\mathrm{Nb}, \mathrm{Ta}, \mathrm{P}$ and Ti anomalies, indicating some subduction component in their genesis. Based on geochemical data, the MMEs are suggested to have been formed by hybridization of basic magma mingled with partially crystallized felsic magma.
\end{abstract}

Keywords: Erenlerdagl, volcanism, Mafic enclaves.

\section{Introduction}

Widespread volcanic activity developed in Turkey during the Neotectonic period, producing volcanic rocks covered an area of about $85,000 \mathrm{~km}^{2}$ in East, Central and West Anatolia (Ketin, 1983). In Central Anatolia, calc-alkaline volcanic units are exposed in large areas situated in the WSW and NW of Konya city (Figure 1), in where transtensive and transpressive tectonic regimes have been effective since the Late Miocene (Kempler and Garfunkel, 1991). The volcanism took place between Late Miocene 11.9 Ma. to Pliocene 3.35 Ma (Keller et al., 1977).

Pre-Mesozoic basement is represented by ophiolitic complex, phyllite, schist, quartzite and dolomi tic limestone, metavolcanic rocks, diorite, diabase, gabbro, peridodite and serpantinite (Özcan ve d iğ., 1990; Eren, 1993; Kurt, 1994). It is unconformably overlain by Upper Miocene-Lower Pliocen e Ulumuhsine formation (Eren, 1993), which is made up by limestone, limestone-mudstone alterna tion and marl. The pyroclastic rocks, which consist of volcanic breccia, agglomerate, tuffite and tuf fs, conformably overlaid the Ulumuhsine formation. The youngest volcanic rocks are andesite, dac ite, and basaltic andesite. Lower Pliocene Yürükler formation overlies unconformably volcanic roc $\mathrm{ks}$, and contains red conglomerate and caliche nodulled mudstone deposits. 


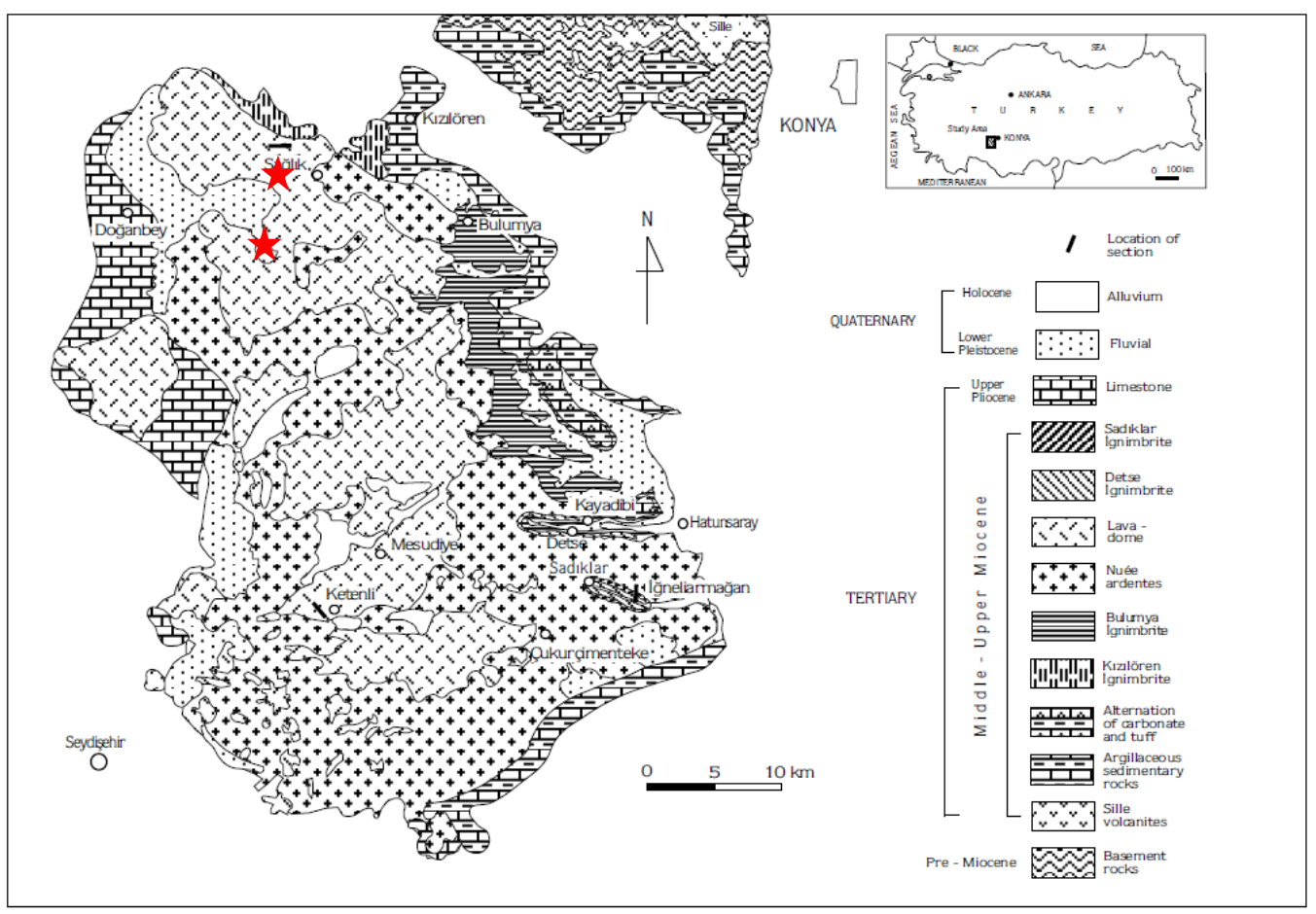

Figure 1 - Location and geological map of the study area (modified from Keller et al., 1977). $\uparrow$. Rock quarries studied.

The study aims to explain the origin of and importance of mafic magmas In Erenlerdagi volcanics. To do so, the study was performed at a rock quarry, in where mafic and felsic magma interactions can be observed very well. The dom contains various mafic microgranular enclaves (MMEs), whic $\mathrm{h}$ have various size (a few $\mathrm{cm}$ to a few meters) and shape (ellipse/sphere to rounded).

\section{Materials and Methods}

The MMEs samples were analyzed to determine contents of major oxides, trace and rare earth elem ents (REE) by ICP-MS at ACME Analytical laboratories in Canada.

\section{Geochemistry}

Bulk rock chemical analyses of the MMEs and three additional MMEs from previous studies (Tem el et al., 1996) are presented in Table 1, with host lava samples (Table 2, Kocak and Zedef, 2016). In an AFM ternary diagram (Figure 2a), the samples clearly define a calc-alkaline trend.

The samples are concentrated on high $\mathrm{K}$ calc-alkaline series (Figure 2b), but some MME samples were also found on calc-alkaline areas in $\mathrm{a} \mathrm{SiO}_{2} \mathrm{vs}_{2} \mathrm{O}$ diagram. In a $\mathrm{Na}_{2} \mathrm{O}+\mathrm{K}_{2} \mathrm{O}$ vs $\mathrm{SiO}_{2}$ diagram, the MMEs samples are situated on mostly basaltic andesite and andesite areas, and a MME sample on basaltic trachy-andesite area while the host rocks are found on dacite and andesite areas (Figure 3a). In Figure 2 and 3a, there is almost no compositional gap between mafic and felsic rocks. In Harker diagram, the $\mathrm{SiO}_{2}$ increases generally with decreasing $\mathrm{TiO}_{2}, \mathrm{FeOt}, \mathrm{MgO}, \mathrm{CaO}$ and $\mathrm{Co}$ (not shown), suggesting fractional crystallization of pyroxene ( \pm olivine), ilmenite and magnetite. Primitive mantle normalized spider trace element diagram of the samples from Sağlık lava domes, the MMEs (Figure 3b) are characterized by an enrichment in large ion litophile elements (LILE), particularly $\mathrm{Cs}, \mathrm{Ba}$ and $\mathrm{Th}$, and depletion in high field strength elements (HFSE). The rocks show progressively decreasing negative $\mathrm{Nb}, \mathrm{Ta}, \mathrm{P}$ and $\mathrm{Ti}$ anomalies, which are typical of subduction- 
related magmas (Pearce, 1983). Chondrite-normalized REE patterns (Figure 4a) for the three rock groups usually exhibit a strongly fractionated REE pattern with high LREE/ HREE for the rocks. In general, all samples are LREE enriched with $(\mathrm{Ce} / \mathrm{Sm})_{\mathrm{N}}=2.54-4.54$, which are similar to those of subduction-related magmas (Pearce 1982, 1983). The more fractionated and LREE-enriched character of the volcanic rocks indicates that the evolution of the rocks involved continental crust (Watters and Pearce, 1987). Plagioclase fractionation is evident from the slight-moderate development of a negative Eu anomaly $\left(\left(\mathrm{Eu} / \mathrm{Eu}^{*}\right)_{\mathrm{N}}:=0.68-0.86\right.$, Figure $\left.4 \mathrm{a}\right)$. The MMEs have les fractionated REE pattern than their hosts. Figure 4 b-c shows that both MMEs and their hosts have mostly volcanic arc geotectonic setting.
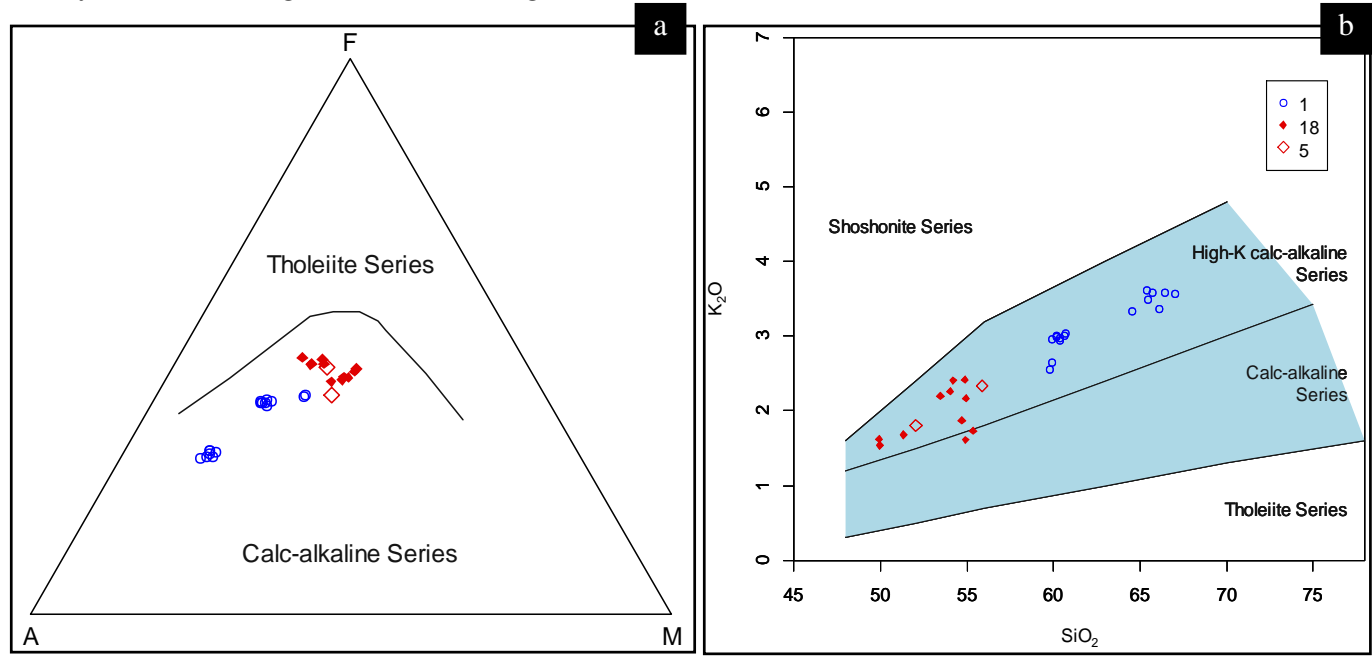

Figure 2 - a) AFM classification of the samples (Irvine and Baragar, 1971), b) $\mathrm{SiO}_{2} \mathrm{vs}_{2} \mathrm{O}$ diagram of the samples (after Peccerillo and Taylor, 1976). Symbols; 1: Sağlık lava domes, 18: MMEs, 5: MMEs (Temel et al., 1996)

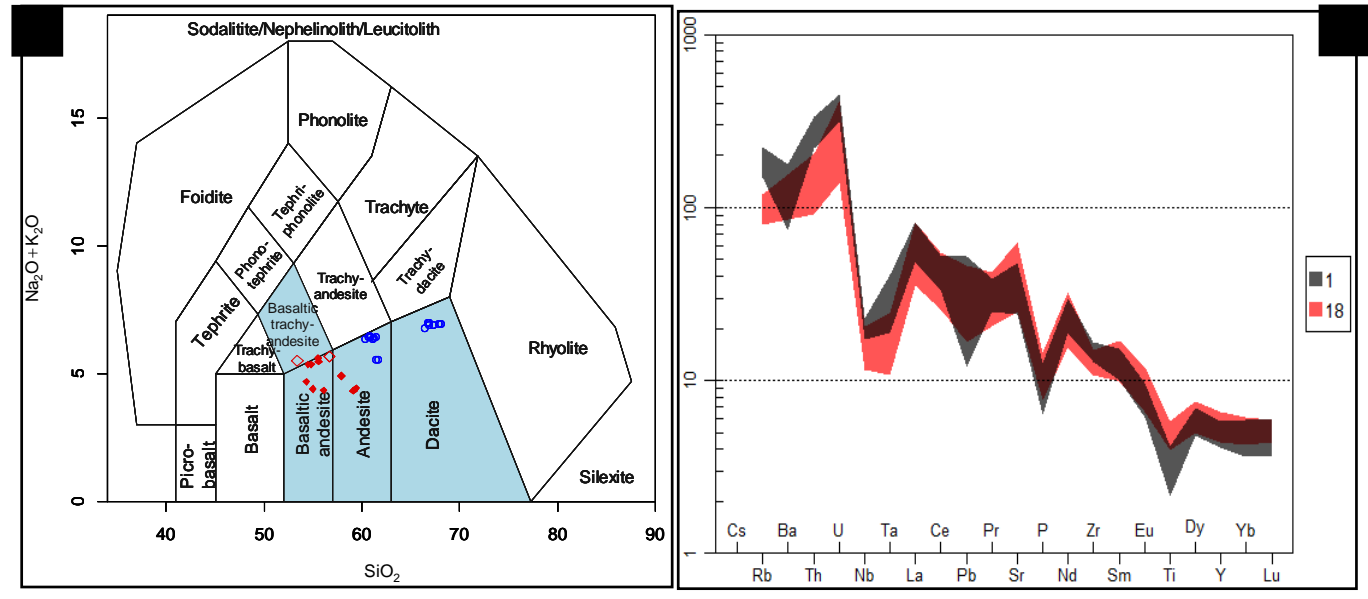

Figure 3 - a) Total alkaline vs $\mathrm{SiO}_{2}$ diagram (Middlemost, 1994) of the samples b) Primitive mantle normalized spider trace element diagram (McDonough and Sun, 1995). Symbols; 1: Sağlık lava domes, 18: MMEs, 5: MMEs (Temel et al., 1998). 
Table 1 - Results of the geochemical analyses of the samples from MMEs.

\begin{tabular}{|c|c|c|c|c|c|c|c|c|c|c|c|c|c|c|}
\hline \multirow{3}{*}{ Samples } & \multirow{3}{*}{$5 \mathrm{~A}$} & \multirow{3}{*}{$\mathbf{8 A}$} & \multirow{3}{*}{$4 \mathrm{~A}$} & \multirow{3}{*}{$42 \mathrm{~A}$} & \multirow{3}{*}{$43 \mathrm{~A}$} & \multirow{3}{*}{$41 \mathrm{~A}$} & \multirow{3}{*}{$1 \mathrm{~A}$} & \multirow{3}{*}{$40 \mathrm{~A}$} & \multirow{3}{*}{$\mathbf{2 A}$} & \multirow{3}{*}{$47 \mathrm{~A}$} & \multirow{3}{*}{ 9A } & \multirow{2}{*}{\multicolumn{3}{|c|}{\begin{tabular}{|l|} 
Temel et al., 1998 \\
KO-3 KO-5 KO-5
\end{tabular}}} \\
\hline & & & & & & & & & & & & & & \\
\hline & & & & & & & & & & & & & & 3 \\
\hline $\mathrm{SiO}_{2}$ & 49.92 & 49.95 & 51.33 & 53.46 & 54.02 & 54.19 & 54.71 & 54.88 & 54.93 & 54.94 & 55.35 & 55.89 & 52.06 & 50.54 \\
\hline $\mathrm{TiO}_{2}$ & 0.81 & 0.8 & 0.9 & 1.17 & 1.14 & 1.11 & 0.93 & 1.11 & 0.89 & 1.13 & 0.87 & 0.83 & 0.86 & 0.76 \\
\hline $\mathrm{Al}_{2} \mathbf{O}_{3}$ & 14.66 & 14.79 & 15.4 & 17.59 & 17.53 & 17.94 & 16.19 & 17.69 & 16.17 & 17.73 & 16.1 & 16.24 & +16.4 & 14.31 \\
\hline $\mathrm{Fe}_{2} \mathrm{O}_{3}$ & 6.23 & 6.2 & 7.22 & 8.54 & 8.37 & 8.16 & 7.4 & 8.18 & 7.1 & 8.08 & 6.69 & 7.33 & 8.41 & 8.61 \\
\hline MgO & 3.5 & 3.65 & 4.24 & 3.79 & 3.9 & 3.08 & 4.31 & 3.49 & 4.2 & 3.44 & 4.01 & 4.57 & 4.13 & 1.09 \\
\hline $\mathrm{CaO}$ & 11.93 & 10.91 & 8.06 & 7.91 & 7.98 & 7.21 & 6 & 7.61 & 5.3 & 7.74 & 5.72 & 7.79 & 9.82 & 9.39 \\
\hline $\mathrm{Na}_{2} \mathrm{O}$ & 2.68 & 2.45 & 2.29 & 3.05 & 3.02 & 3.05 & 2.76 & 3.12 & 2.41 & 3.23 & 2.38 & 3.23 & 3.54 & 2.2 \\
\hline $\mathbf{K}_{2} \mathbf{O}$ & 1.62 & 1.54 & 1.68 & 2.2 & 2.26 & 2.41 & 1.87 & 2.42 & 1.61 & 2.17 & 1.73 & 2.34 & 1.81 & 1.31 \\
\hline $\mathrm{P}_{2} \mathrm{O}_{5}$ & $\mid 0.17$ & 0.16 & 0.2 & 0.28 & 0.27 & 0.3 & 0.16 & 0.27 & 0.17 & 0.29 & 0.17 & 0.27 & 0.27 & 0.24 \\
\hline MnO & 0.37 & 0.4 & 0.23 & 0.13 & 0.13 & 0.13 & 0.18 & 0.12 & 0.18 & 0.13 & 0.15 & 0.123 & 30.193 & 0.17 \\
\hline LOI & 7.8 & 8.9 & 8.2 & 1.5 & 0.9 & 2 & 5.2 & 0.7 & 6.8 & 0.7 & 6.5 & 0.98 & 1.87 & 0.83 \\
\hline Sum & 99.74 & 99.75 & 99.72 & 99.58 & 99.55 & 99.58 & 99.71 & 99.58 & 99.73 & 99.58 & 99.73 & 99.59 & 999.36 & 99.25 \\
\hline $\mathbf{N i}$ & 2.2 & 1.4 & 2.3 & 2.6 & 2.9 & 2.2 & 2.9 & 2.4 & 2.8 & 2.1 & 2.4 & $\mid 10.8$ & 19.1 & 184.5 \\
\hline Co & 14.4 & 13.6 & 15.8 & 21.9 & 21.2 & 20.6 & 16 & 21.3 & 15.5 & 20.1 & 15.2 & 34.4 & 27.6 & 46.9 \\
\hline $\mathbf{P b}$ & 4.4 & 2.7 & 5.5 & 2.9 & 2.6 & 2.9 & 5.4 & 2.5 & 6.1 & 2.9 & 6.9 & & & \\
\hline Rb & 57.3 & 47.9 & 57.2 & 62.2 & 65 & 70.5 & 66.6 & 71 & 50.4 & 63.5 & 52.2 & 62.2 & 51.5 & 25.9 \\
\hline $\mathrm{Sr}$ & 615.3 & 548.6 & 577.1 & 1213 & 1221 & 1222 & 635.5 & 1207 & 495.9 & 1249 & 541.5 & 1195 & 637.7 & 1162 \\
\hline Hf & 3.6 & 2.4 & 3.2 & 4.1 & 3.6 & 4.8 & 3.2 & 3.9 & 3.3 & 4.3 & 3.6 & & & \\
\hline Nb & 8.7 & 7.6 & 9.1 & 11.6 & 11.8 & 11.9 & 10.2 & 10.3 & 8.4 & 13.6 & 8.1 & 9.2 & 14.7 & 5.3 \\
\hline Ta & 0.7 & 0.4 & 0.5 & 0.5 & 0.6 & 0.7 & 0.5 & 0.9 & 0.5 & 0.8 & 0.6 & & & \\
\hline Th & 8.5 & 7.5 & 7.3 & 14.4 & 16.2 & 16.4 & 8 & 15.7 & 8.9 & 16.2 & 9 & & & \\
\hline $\mathbf{U}$ & 8.3 & 3 & 3.5 & 3.8 & 5 & 4.5 & 4.3 & 4.5 & 2.8 & 5.4 & 2.8 & 175.5 & 5153.9 & 164.4 \\
\hline $\mathbf{Z r}$ & 128.7 & 113.3 & 125.2 & 148.7 & 152.7 & 156.5 & 116.8 & 149.2 & 115 & 151.7 & 124 & 150 & 113.5 & 127.6 \\
\hline $\mathbf{Y}$ & 23.3 & 20.3 & 24.2 & 24.9 & 25.1 & 28.4 & 23.2 & 22.8 & 18.9 & 23.8 & 20.2 & |21.1 & 30.5 & 16.4 \\
\hline La & 25.8 & 24.6 & 28.4 & 47.3 & 49.1 & 53.7 & 25.7 & 46.2 & 22.8 & 49.2 & 25.4 & & & \\
\hline $\mathrm{Ce}$ & 46.6 & 43.6 & 53.2 & 81.8 & 86.8 & 91.4 & 50 & 85.3 & 45.9 & 87.4 & 48.1 & & & \\
\hline Pr & 5.39 & 5.25 & 6.29 & 9.8 & 10.11 & 10.73 & 5.87 & 9.82 & 5.25 & 10.28 & 5.66 & & & \\
\hline Nd & 22.7 & 23.3 & 26.6 & 40.6 & 39.7 & 38.7 & 21.6 & 32 & 19.3 & 37.9 & 23.2 & & & \\
\hline Sm & 4.12 & 4.01 & 4.84 & 6.68 & 6.87 & 6.76 & 4.62 & 6.66 & 4.27 & 6.73 & 4.57 & & & \\
\hline Eu & 1.11 & 1.04 & 1.27 & 1.67 & 1.59 & 1.83 & 1.26 & 1.71 & 1.11 & 1.65 & 1.01 & & & \\
\hline Gd & 3.99 & 3.58 & 4.68 & 5.36 & 6.09 & 6.19 & 4.4 & 5.63 & 3.89 & 5.65 & 4.48 & & & \\
\hline $\mathbf{T b}$ & 0.63 & 0.6 & 0.72 & 0.82 & 0.82 & 0.83 & 0.69 & 0.81 & 0.59 & 0.81 & 0.65 & & & \\
\hline Dy & 4.09 & 3.52 & 4.28 & 4.23 & 4.47 & 5.09 & 4.04 & 4.23 & 3.37 & 4.16 & 3.76 & & & \\
\hline Ho & 0.79 & 0.79 & 0.91 & 0.89 & 0.8 & 1 & 0.89 & 0.92 & 0.71 & 0.82 & 0.72 & & & \\
\hline Er & 2.28 & 2.14 & 2.62 & 2.68 & 2.82 & 2.96 & 2.61 & 2.49 & 2.13 & 2.52 & 2.33 & & & \\
\hline $\mathbf{T m}$ & 0.3 & 0.29 & 0.34 & 0.39 & 0.36 & 0.39 & 0.42 & 0.4 & 0.32 & 0.35 & 0.31 & & & \\
\hline Yb & 2.13 & 1.87 & 2.34 & 2.38 & 2.64 & 2.26 & 2.6 & 2.36 & 2.09 & 2.68 & 2.17 & & & \\
\hline Lu & 0.38 & 0.3 & 0.35 & 0.34 & 0.35 & 0.33 & 0.4 & 0.32 & 0.32 & 0.35 & 0.34 & & & \\
\hline
\end{tabular}


Table 2 - Results of the geochemical analyses of the samples from the lava domes (Kocak and Zedef, 2016)

\begin{tabular}{|c|c|c|c|c|c|c|c|c|c|c|c|c|c|c|c|c|}
\hline & & $2 C$ & 3B & $\bar{B}$ & 5B & $\overline{7 B}$ & $\bar{B}$ & 9C & $\overline{1 B}$ & DB & $41 B$ & $\overline{2 B}$ & 43B & $\overline{4 B}$ & $46 B$ & $47 B$ \\
\hline 22 & 5.11 & .9 & 7 & .57 & $03 . / 5$ & 565.44 & & 9.76 & & .10 & & 0.6 & & 0. & 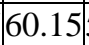 & 59. \\
\hline & .45 & 8 & 45 & .47 & 77 & 0.47 & 48 & 0.83 & 0.43 & 0.8 & & 0.79 & 0.78 & 0.79 & 0.8 & \\
\hline 203 & .52 & 15.91 & & 43 & & 5.15 & & 07 & & 6.94 & & 56 & & 16.8 & $T$ & \\
\hline 20 & 3.76 & 6.3 & 68 & 3.74 & 3.78 & 3.84 & 3.95 & 6.27 & 3.57 & 6.11 & 5.99 & 5.95 & 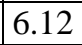 & 6.04 & 5.01 & \\
\hline & 61 & 37 & & 34 & 17 & 56 & & 33 & & 54 & & .39 & & 38 & 61 & \\
\hline & 73 & 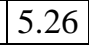 & & 73 & 4.1 & 4.48 & 01 & 38 & & 93 & 5.77 & 5.68 & & 77 & 78 & \\
\hline & 39 & 73 & & 22 & 27 & 26 & 23 & 84 & 19 & 39 & 39 & 3.3 & 33 & 3.33 & 3.4 & \\
\hline & 3.37 & 54 & 57 & 34 & 58 & 3.49 & 3.62 & 56 & 3.58 & 99 & 2.97 & 3.04 & 2.94 & 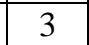 & 01 & \\
\hline & 17 & & 6 & 7 & 18 & 18 & 18 & 16 & 16 & 25 & 24 & 24 & 10 & 0.26 & 25 & \\
\hline & 08 & 13 & 07 & 0.1 & 08 & 0.1 & 0.1 & 0.12 & 0.07 & .11 & .11 & 0.1 & 11 & 0.11 & 11 & 0 . \\
\hline & 1.6 & 2.6 & 1.3 & 2.4 & 1.4 & 1.8 & 1.8 & 2.4 & 1.8 & 0.4 & .5 & 0.9 & .8 & 0.5 & .6 & \\
\hline Sun & .75 & .7 & .8 & & & & & & & 9. & & 9. &. & 9. & . & 9.6 \\
\hline $\mathbf{N i}$ & 3.4 & 2.1 & 3.2 & 3 & 3.4 & 3.2 & 3.5 & 2.6 & 3.2 & 2 & 1.9 & 2.4 & 2.6 & 2.3 & 1.8 & 1.9 \\
\hline C & 6.7 & 2.2 & .7 & 8.7 & 8. & 7.3 & 7.7 & 14.3 & & 14.6 & 13.7 & 12.8 & 14 & 14.7 & 3.8 & 14 \\
\hline H & & & 4.1 & 3.5 & 4.7 & 4.1 & 4.8 & 4.3 & 4.2 & 3.9 & & 4.5 & 4 & 5.2 & 5.2 & 4.1 \\
\hline $\mathbf{N}$ & & 2.8 & .6 & 14.1 & 15 & 14.1 & 3.4 & 12.2 & 13.3 & 2.5 & .5 & 3.1 & .1 & 2.6 & 3 & 11.4 \\
\hline $\mathbf{R}$ & 5.9 & 3.4 & 0.5 & 15. & 31. & 22. & 130.4 & 99.6 & 35.3 & 2.3 & 1.9 & 5.5 & 4.4 & 96.9 & 93 & 90 \\
\hline B & 24 & 490 & 1027 & 984 & 1015 & 980 & 988 & 621 & 981 & 1151 & 1103 & 1055 & 1120 & 1176 & 113 & 108 \\
\hline & 529.2 & 524.9 & 501.8 & 3514.5 & 503.7 & 541 & 479. & 77.4 & 518.8 & 949.5 & 906.7 & 858.2 & 906.4 & 878 & 900. & 925 \\
\hline $\mathbf{P}$ & 32 & 5.4 & 3.5 & 2.4 & 2.8 & 3.5 & 2.5 & 5 & 7.8 & 2.3 & 1.9 & 1.8 & 1.8 & 1.9 & 2 & 2.1 \\
\hline n & 27 & 35 & 23 & 30 & 31 & 25 & 26 & 39 & 35 & 19 & 15 & 17 & 20 & 18 & 16 & 14 \\
\hline$T$ & & 1 & 1.5 & 1.1 & 1.4 & 1.2 & 1.3 & 1 & 1.2 & 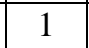 & 0.7 & 1.2 & 1 & 1 & 0.9 & 0.9 \\
\hline $\mathbf{1}$ & 3 & 20.4 & 8.8 & 23.7 & 26.7 & 24.4 & 24.6 & 17.7 & 25.7 & 21.2 & 21.3 & 20.7 & 22 & 22.3 & 1.3 & 20.7 \\
\hline$\overline{\mathbf{U}}$ & 1.1 & 6.3 & 8.8 & 7.7 & 7 & 8.9 & 9 & 7.3 & 8.1 & 6.6 & 6.3 & 6.3 & 6.2 & 6.5 & 6.5 & 6.4 \\
\hline $\mathbf{Z r}$ & 151.2 & 133. & 166.7 & 43. & 68.6 & 55. & 70. & 46.1 & 57. & 73. & 168. & 64. & 174 & 75. & 170. & 164 \\
\hline $\bar{Y}$ & .5 & 21.2 & .5 & 18.6 & \begin{tabular}{|l|}
19.1 \\
\end{tabular} & 18.2 & 18.6 & 23.2 & \begin{tabular}{|l|}
17.8 \\
\end{tabular} & 23.9 & 24.9 & 21.6 & 22.1 & 25.1 & 21.9 & 22.8 \\
\hline L & .5 & 31.6 & 6.9 & 8 & 46.6 & H. 1 & 41.4 & 32 & 42.1 & 9.1 & 51.4 & 46 & 49 & 53 & 51.4 & 49. \\
\hline & 1.5 & 56.7 & 0.9 & 2 & 82 & 5.9 & 72 & 3.8 & 73 & 86.4 & 84.6 & 83.1 & 84.4 & 86.7 & 88.4 & 85 \\
\hline & & 6.32 & 32 & 83 & 8.47 & 7.96 & 7.56 & 6.49 & 7.38 & 9.6 & 9.35 & & 9.26 & 9.75 & .51 & 9.4 \\
\hline No & 36 & 3.7 & 8 & 26.9 & 9.8 & 26.8 & 25.8 & 3.9 & 27.3 & 37.1 & 33.8 & 34.6 & 34.1 & 35.5 & 5.5 & 36. \\
\hline S1 & & 25 & 18 & 41 & 79 & 4.09 & 4.7 & 4.48 & 4.2 & .94 & 5.54 & \begin{tabular}{|l}
5.71 \\
\end{tabular} & 78 & 6.16 & .82 & 5.69 \\
\hline $\mathrm{CI}$ & & 1. & 97 & 0.97 & 03 & 0.96 & 0.97 & 02 & 0.95 & 1.42 & 37 & .38 & 42 & 1.49 & 45 & 1.4 \\
\hline$G$ & & 4.07 & 4.22 & 4 & 49 & 3.86 & 4.09 & 32 & 3.64 & & 5.07 & 5 & .3 & .22 & 5.08 & 5.08 \\
\hline $\mathbf{T t}$ & 54 & 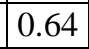 & .59 & 66 & 0.6 & 0.57 & 0.58 & 68 & 0.53 & .7 & 0.7 & 67 & .72 & .77 & .7 & 0.73 \\
\hline D & 3.3 & 3.71 & 76 & 3.46 & .21 & 3.43 & 3.47 & 18 & 3.3 & 3.84 & 3.93 & 15 & 3.58 & 4.68 & .62 & 3.7 \\
\hline & 0 . & 38 & 58 & 56 & 65 & 0.64 & 0.68 & 0.84 & 0.56 & 0.8 & 0.86 & 0.69 & 0.79 & 0.98 & .81 & 0.77 \\
\hline & 96 & 2 & 3 & 2 & 1.91 & 1.74 & 1.92 & 2.63 & 1.91 & 2.23 & 2.74 & 1.98 & 2.29 & 2.36 & 2.49 & 2.42 \\
\hline & 0 & 0 & & 0.29 & 0.29 & 0.34 & 0.3 & 0.33 & 0.26 & .31 & 0.37 & 0.31 & 0.36 & 0.38 & 0.32 & 0.36 \\
\hline & & 2. & 1.99 & 9 & 87 & 1.88 & 1.77 & 2.12 & 2 & 2.31 & 1.91 & 2.23 & 2.21 & 2.59 & 2.23 & 2.3 \\
\hline & 29 & 41 & 33 & 0.31 & 33 & 0.25 & 0.28 & 0.39 & 25 & \begin{tabular}{|l|} 
\\
\end{tabular} & 033 & 0.3 & 0.3 & \begin{tabular}{|l|} 
\\
\end{tabular} & 0.3 & 0.3 \\
\hline
\end{tabular}




\section{Discussions and Conclusions}

The MMEs have relatively low $\mathrm{SiO}_{2}$ content (50-56\%) and intermediate to high molar Mg\# (20-55), which is inconsistent with partial melting of the mafic lower crustal rocks, and requires a mantlederived component. As a whole, existence of colinear variations in Harker diagrams and minor Eu anomaly indicate that fractional crystallization was substantial in the petrogenesis of the Erenlerdagi Volcanites. In contrast, in $\mathrm{A} / \mathrm{CNK}$ and $\mathrm{Y}$ vs $\mathrm{SiO}_{2}$ diagrams (Figure 5) two distinct group appears, which is evident also in Lu vs La, vs \#mg diagrams (not shown). Existence of MMEs suggests maficfelsic interaction and mingling (Barbarin and Didier, 1992; Kocak, 2006) by injection hot mafic magma injected into felsic magma. Accordingly, Temel et al. (1998) suggest a mix of mantle and crustal sources for the Erenlerdag1 volcanites based on an isotopic study.

The Konya volcanites are characterized by intermediate to high $\mathrm{K}_{2} \mathrm{O}$ (1.31-3.62 wt.\%), Rb (26-135 ppm), $\mathrm{Ba}(490-1176 \mathrm{ppm}), \mathrm{K}_{2} \mathrm{O} / \mathrm{Na}_{2} \mathrm{O}$ (0.5-1.1) and $\mathrm{FeOt} / \mathrm{MgO}$ (1.44-7.11) ratios, which is similar to Andean type andesites series formed in relation with subduction event, which is evident in some geotectonic setting diagrams. It is likely that the volcanics may have been produced by assimilationfractional crystallisation process in relation with the collision of Eurasian and Arabian plates.

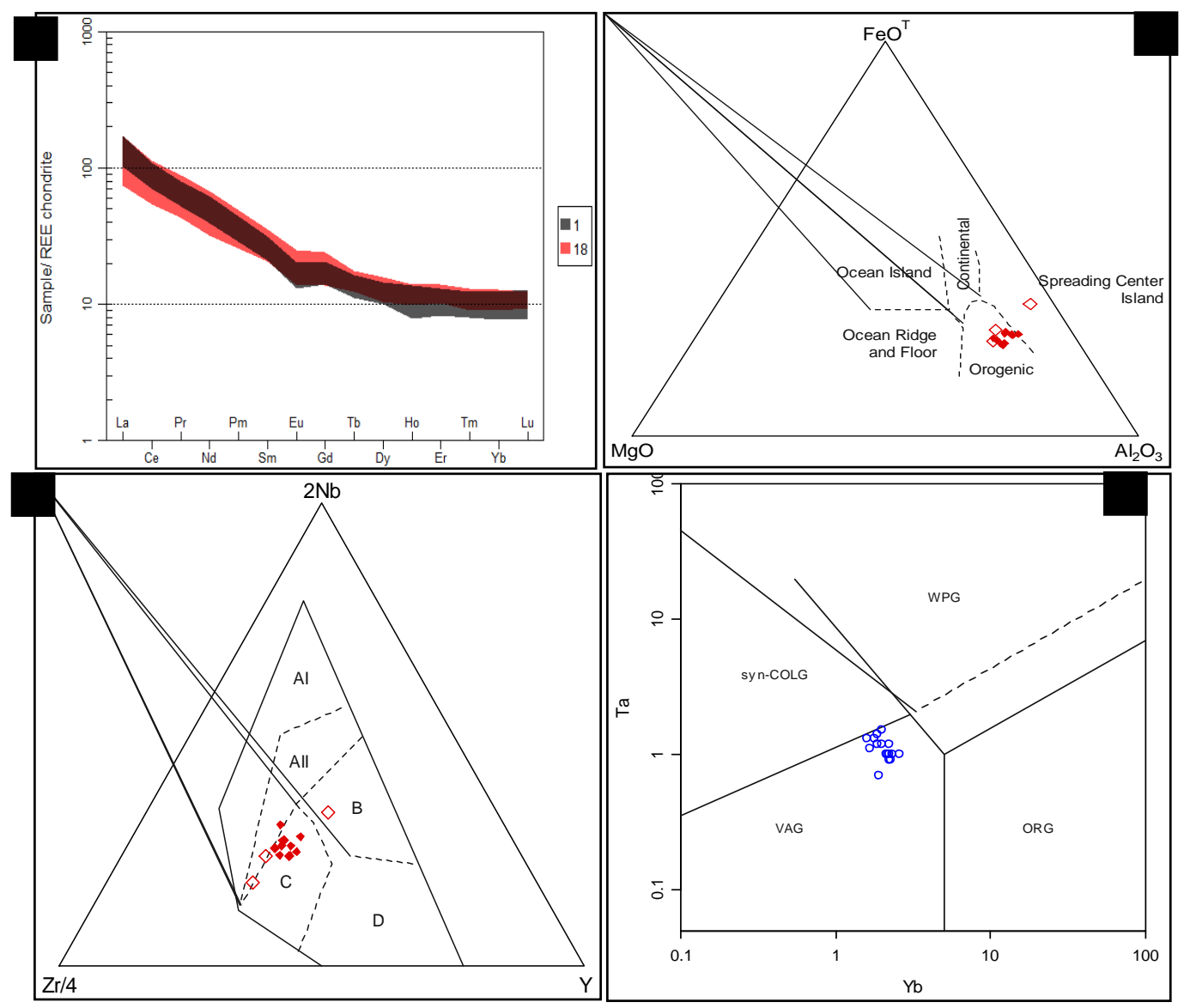

Figure 4 - a) Chondrite normalized REE pattern of the Sağlık lava domes (1), Takkeli tepevolcanics (2) and sill (3). Normalized values are from Boynton, 1984, b)FeOt-MgO- $\mathrm{Al}_{2} \mathrm{O}_{3}$ ternary diagram (Pearce et al.,1977), c) Zr/4-Y-2Nb (Meschede, 1986) (samples with high SiO2(>55) are excluded). AI-II: WP alkaline, AII-C: WP Tholeiitic, B: E-MORB, D: NMORB, C-D: VAB, d) Ta vs Yb diagram (after Pearce et al., 1984). Symbols; 1: Sağlık lava domes, 18: MMEs, 5: MMEs (Temel et al., 1996). 

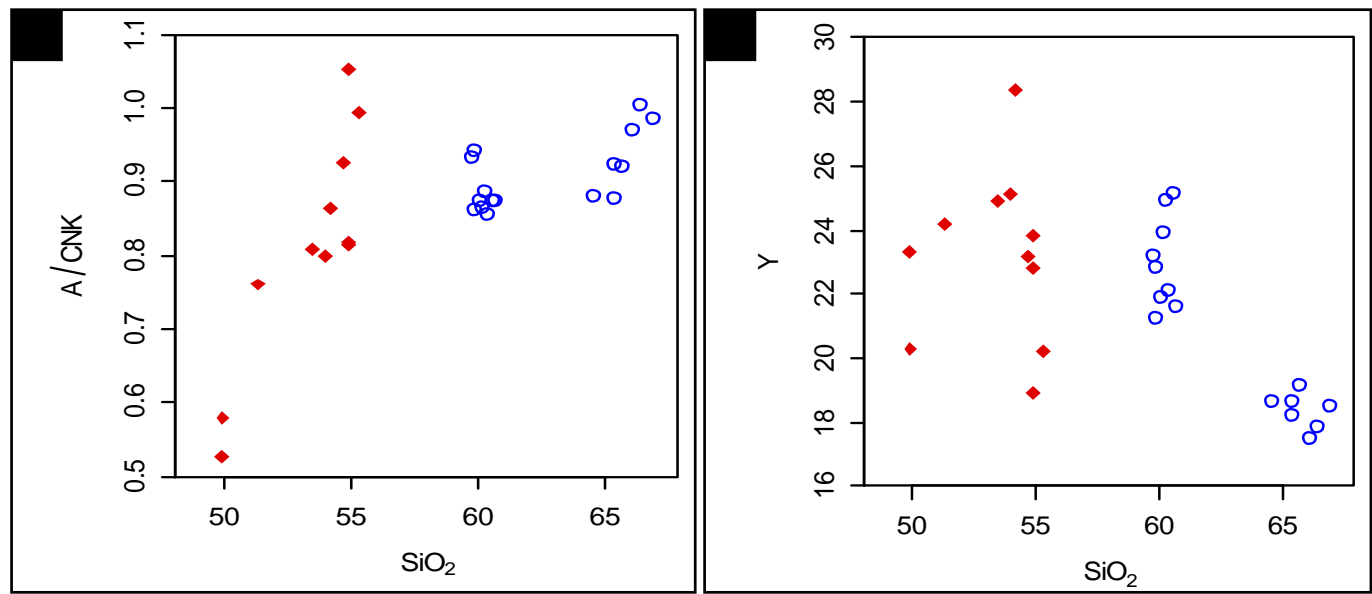

Figure 5 - a)A/CNK vs $\mathrm{SiO}_{2}$, b) Y vs $\mathrm{SiO}_{2}$. Symbols; 1: Sağlık lava domes, 18: MMEs, 5: MMEs (Temel et al., 1996).

\section{Acknowledgements}

Thanks to Selcuk University (BAP, Konya/Turkey) Research Fund for the financial support of the study.

\section{References}

Temel, A., Gundogdu, M.N. and Gourgaud, A., 1998. Petrological and geochemical characteristics of cenozoic high-K calc-alkaline volcanism in Konya, central Anatolia, Turkey, Journal of Volcanology and Geothermal Research, 85, 327-354.

Barbarin, B. and Didier, J., 1992. Genesis and Evolution of Mafic Microgranular Enclaves through Various Types of Interaction between Coexisting Felsic and Mafic Magmas, Transactions of the Royal Society of Edinburgh-Earth Sciences, 83,145-153.

Boynton, W.V., 1984. Cosmochemistry of the rare earth elements: meteorite studies. In: Henderson, P., ed., Rare earth elements, Elsevier, 63-114.

Eren, Y., 1993. Eldes - Derbent - Tepeköy - Söğüttözü (Konya) arasinin jeolojisi, Doktora Tezi, 5, Fen Bilimleri Enstitüsü, Konya, 224 pp. (yayinlanmamis).

Irvine, T.N. and Baragar, W.R., 1971. A guide to the chemical classification of the common igneous rocks, Canadian Journal of Earth Sciences, 8, 523-548.

Keller, J., Jung, D., Burgath, K. and Wolf, F., 1977. Geologie und petrologie des Neogenen kalkalkali-vulkanismus von Konya (Erenler Dağ-Alaca Dağ-Massiv Zentral-Anatolian), Geo. Jb. B., 25, 37-117.

Kempler D. and Garfunkel, Z., 1991. The northeast Mediterranean triple junction from a plate kinematics point of view, Bull. Tech. Univ., Istanbul, 44, 203-232.

Ketin, İ., 1983, Türkiye Jeolojisine Genel Bir Bakış Istanbul Technical Univercity, Publications, Istanbul, 595 pp.

Kocak, K., 2006. Hybridization of mafic microgranular enclaves: mineral and whole-rock chemistry evidence from the Karamadazi Granitoid, Central Turkey, International Journal of Earth Sciences, 95(4), 587-607.

Kocak, K. and Zedef, 2016. Geochemical characteristics of the lava domes in Yatagan village and Sağlik town, from Erenlerdagi (Konya, Central Turkey) volcanites, Acta Geobalcanica, 2(1), 7-19.

Kurt, H.K., Özkan, A.M. and Kocak, K., 2003. Geology, Petrography and Geochemistry of the Subduction Related Volcanic Rocks, West of Konya, Central Anatolia, Geological Bulletin of Turkey, 46(2), 39-51. 
Kurt, H., 1994. Petrography and geochemistry of Kadinhani (Konya) area, Central Turkey, PhD th esis, Glasgow University (unpublished), UK, $191 \mathrm{pp}$.

McDonough, W.F. and Sun, S.-S., 1995. Composition of the Earth, Chemical Geology, 120, 223253.

Meschede, M., 1986. A method of discriminating between different types of mid-ocean ridge basal ts and continental tholeiites with the Nb-Zr-Y diagram, Chemical Geology, 56, 207-208.

Middlemost, E.A.K., 1994. Naming materials in the magma/igneous rock system, Earth Science Reviews, 37(1), 215-224.

Özcan, A., Göncüoglu, M.C., Turhan, N., Sentürk, K., Uysal, S. and ve Isık, A., 1990. Konya- Kad ınhanı-Ilgın dolayının temel jeolojisi, M.T.A. Rapor, No: 9535 (in Turkish, unpublished).

Pearce, T.H., Gorman, B.E. and Birkett, T.C., 1977. The relationship between major element chemistry and tectonic environment of basic and intermediate volcanic rock, Earth and Planetary Science Letters, 36, 121-132.

Pearce, J.A., 1982. Trace element characteristics of lavas from destructive plate boundaries; 525548, In: Thorp, R.S., ed., Andesites: Orogenic Andesites and Related Rocks, John Wiley and Sons, New York, 724 pp.

Pearce, T.H., Gorman, B.E. and Birkett, T.C., 1977. The relationship between major element chemistry and tectonic environment of basic and intermediate volcanic rock, Earth and Planetary Science Letters, 36, 121-132.

Pearce, J.A., Harris, N.B.W. and Tindle, A.G., 1984. Trace element discrimination diagrams for the tectonic interpretation of granitic rocks, Journal of Petrology, 25, 956-983.

Pearce, J.A., 1983. Role of the sub-continental lithosphere in magma genesis at acrive continental margins: p. 230-249. In: Hawkesworth, C.J. and Norry, M.J., eds., Continental Basalts and Mantle Xenoliths, Shiva Publishing Ltd., Cambridge, Mass., 272 pp.

Peccerillo, A. and Taylor, S.R., 1976. Geochemistry of Eocene calcalkaline volcanic rocks from the Kastamonu area, northern Turkey, Contrib Mineral Petrol, 58, 63-81.

Temel, A., Gündoğdu, M.N. and Gourgaud, A., 1998. Petrological and geochemical characteristics of Cenozoic high-K calc-alkaline volcanism in Konya, Central Anatolia, Turkey, J. Volcanol. Geotherm. Res, 85, 327-354.

Watters, B.R. and Pearce, J.A., 1987. Metavolcanic rocks of La Ronge domain in the Churchill Province, Saskatchewan: geochemical evidence for a volcanic arc origin, Geol. Soc. Lond., 33, 167-182. 